\title{
Identification of BoLA DRB3.2 Alleles Present in White Fulani and Muturu Cattle Breeds
}

\author{
Ridwan 0. Ahmed ${ }^{1 *}$, Semiu F. Bello², Matthew J. Hegarty ${ }^{1}$ \\ ${ }^{1}$ Institute of Biological, Environmental and Rural Science, Aberystwyth University, Aberystwyth, UK \\ ${ }^{2}$ Department of Animal Genetics, Breeding and Reproduction, College of Animal Science, South China Agricultural University, \\ Guangzhou, China \\ Email: *ahmedridwan341@gmail.com
}

How to cite this paper: Ahmed, R.O., Bello, S.F. and Hegarty, M.J. (2020) Identification of BoLA DRB3.2 Alleles Present in White Fulani and Muturu Cattle Breeds. Open Journal of Animal Sciences, 10, 725-734. https://doi.org/10.4236/ojas.2020.104047

Received: September 15, 2020

Accepted: October 19, 2020

Published: October 22, 2020

Copyright (c) 2020 by author(s) and Scientific Research Publishing Inc. This work is licensed under the Creative Commons Attribution International License (CC BY 4.0).

http://creativecommons.org/licenses/by/4.0/

\begin{abstract}
Cattle production is an important aspect of animal agriculture. Disease predisposition in cattle can lead to lowered productivity and poor animal welfare. To select and breed for the fittest cattle population, it is expedient that we understand the genetic basis of disease tolerance/resistance. Major histocompatibility complex (MHC) is a vital component of the immune system in vertebrates. Its genes are crucial determinants for immune response and resistance to infectious diseases. The bovine MHC is referred to as Bovine Lymphocyte antigen (BoLA) with its most functional and highly variable region located in the exon 2 (BoLA-DRB3.2). Over 100 alleles of BoLA-DRB3.2 have been identified in cattle and many studies have associated polymorphism in this region with disease resistance/susceptibility. In this study, we investigated the polymorphic nature of BoLA-DRB3.2 in the White Fulani and Muturu cattle breeds using a single PCR-sequence based typing. We identified 26 and 25 alleles in White Fulani and Muturu breeds, respectively, with only six alleles being mutual in the two breeds. Some of the alleles identified in this study have been noted as markers for disease status in cattle. $B o L A-D R B 3^{*} 014: 01: 01, B o L A-D R B 3^{*} 011: 01$, and $B o L A-D R B 3^{*} 008: 01$ alleles have been associated with Bovine leukemia virus (BLV) resistance in cattle. BoLA-DRB3 ${ }^{*} 014: 01: 01, B o L A-D R B 3^{*} 001: 01$, and BoLA-DRB3 ${ }^{*} 011: 01$ were linked with mastitis resistance in Japanese Holstein cows. While no inference can be drawn in terms of association with disease status, this study confirms the highly polymorphic and diverse nature of BoLA-DRB3 in White Fulani and Muturu cattle breeds.
\end{abstract}

\section{Keywords}

Major Histocompatibility Complex, Bovine Lymphocyte Antigen (BoLA), Polymorphism, White Fulani, Muturu 


\section{Introduction}

Cattle production is a key aspect of livestock agriculture with its output serving as valuable protein source for consumers as well as providing financial benefits for farmers. Challenges posed by diseases can lead to lowered productivity, poor animal welfare and impose huge financial burden on farmers. It is expedient that we understand the genetic basis of disease tolerance/resistance in order to select and breed for the most tolerant or resistant cattle population. Major histocompatibility complex (MHC) is a vital component of the immune system in vertebrates [1]. MHC genes are crucial determinants for immune response and resistance to infectious diseases [2]. The bovine MHC is referred to as Bovine Lymphocyte antigen (BoLA) [3], and it is mapped to BTA 23 [4]. It has two broad divisions-class I and II. The class II region is further sub-divided into class IIa and class IIb [5]. The genes in the MHC class IIa subregion encode either the DQ or DR molecule. DRA genes which encode the $\alpha$-chain of the DR molecule show less polymorphism in comparison with the gene encoding the $\beta$-chain (DRB) [5]. Of the three bovine DRB loci, only $D R B 3$ is functional [6]; and within $D R B 3$, most variation occurs in the exon 2 region [7] [8]. DRB3 is reported to influence the strength and specificity of antigen-specific T-cell response to infection [9]. Polymorphism in the $B O L A-D R B 3$ region has been linked to differences observed in individual cattle in their response to pathogenic diseases [5]. Over 100 alleles of BoLA-DRB3.2 have been reported [10]. As a result of its role in encoding for the $\beta 1$ domain of the only widely expressed $D R B$ gene in cattle (DRB3) and its high variability, many studies have investigated polymorphism in the BoLA-DRB3 exon 2 (BoLA-DRB3.2), and its association with disease resistance in cattle [11] [12] [13]. In addition to disease status, BoLA-DRB3.2 polymorphism has also been associated with milk production traits [14] [15].

Using PCR-sequence based typing, Takeshima et al. [16] investigated the level of polymorphism and genetic diversity of BoLA.DRB3.2 in Philippine native cattle breeds. They identified 83 BoLA.DRB3.2 alleles in 1120 cattle; with five of the alleles being novel. The researchers also observed that in comparison with breeds from other countries, native Philippine cattle possessed a higher number of alleles (71 vs. 35), making them more polymorphic and diverse. By constructing a phylogenetic tree, the authors concluded that there is a distinction between cattle coming from the northern and southern Philippine. Carignano et al. [17] reported that inter-animal genetic variation in BOLA.DRB3.2 is associated with the level of bovine leukaemia virus infection in Holstein and Holstein $\mathrm{x}$ Jersey crossbreed. The outcome of the study showed that $D R B 3^{*} 0902$ allele was associated with lower infection load, while two alleles $\left(D R B 3^{*} 1001\right.$ and $\left.D R B 3^{*} 1201\right)$ were associated with a high level of infection. Juliarena et al. [13] and Juliarena et al. [18] had earlier associated DRB3.2 alleles with bovine leukaemia virus infection levels but cautioned that, despite high association observed between $D R B 3^{*} 0902$ allele and low bovine leukaemia infection level, it is not enough to make a definite conclusion that this genetic variation is solely responsible for the 
phenotypic difference observed in cattle. The authors opined that a complex genetic and epigenetic interaction is involved in the regulation of bovine leukaemia virus infection.

There is a dearth of information on the extent of BOLA-DRB3.2 polymorphism in White Fulani and Muturu breeds. The White Fulani, a Bos indicus, is regarded as the most numerous and widespread Nigerian cattle breed representing $37 \%$ of the country's herd while Muturu, a West African taurine, is small-bodied trypanotolerant cattle [19]. The aim of this study was to investigate the polymorphic nature of BoLA-DRB3.2 in the White Fulani and Muturu breeds using a single PCR-sequence based typing.

\section{Materials and Methods}

\section{Data collection and Animal source}

The cattle population used for this study are those described by Ahmed et al. [19]. Briefly, it consisted of 80 cattle, forty from each of White Fulani and Muturu breed reared under a semi-intensive production system. Using the PG-100 collection kit from PERFORMAGENE, nasal swab was collected from each animal for DNA extraction.

\section{Genomic DNA Extraction}

Genomic DNA was extracted from the nasal swab as described by Ahmed et al. [19].

\section{PCR amplification and DNA sequencing}

280-bp covering all exon 2 of BoLA-DRB3 was amplified in a single PCR [20] using DRB3FWR (CGC TCC TGT GA (C/T) CAG ATC TAT CC) and DRB3REV (CAC CCC CGC GCT CAC C) primers as described by Miltiadou et al. [21]. Primer processing, PCR conditions and protocols are as described by Ahmed et al. [19] except for the $59^{\circ} \mathrm{C}$ annealing temperature used here. PCR products were checked on 1\% agarose gel. PCR amplicon was purified prior to sequencing (removal of contaminants and primers) as described by Ahmed et al. [19]. Sequencing reaction was performed at the IBERS Gogerddan Sequencing Facility primed with the DRB3FRW primer to obtain forward sequences.

Sequence-based typing was used to identify $D R B 3.2$ alleles present in the cattle population examined. BoLA-DRB3.2 allele database, which has all previously identified alleles and sequences, was downloaded from the IPD-MHC website (https://www.ebi.ac.uk/ipd/mhc/group/BoLA). Using Genomic Workbench (CLC Bio Ltd, version 6.0), a custom BLAST database was created for all downloaded BoLA-DRB3 alleles. BLAST analysis of the amplified 280 bp sequence products against the created database was used to detect BoLA-DRB3.2 alleles present in White Fulani and Muturu cattle population. For individual animal sequence searched against the database, BoLA-DRB3.2 allele with the highest percentage identity to the query sequence was selected. Appendix shows the percentage identity to the reference sequence, for all the 78 sequences examined in this study, with averages of $96.74 \%$ and $97.48 \%$ in White Fulani and Muturu, 
respectively. The hit length for each sequence is also included in Appendix.

\section{Results}

Presented in Table 1 are the BoLA-DRB3.2 alleles identified and their frequencies of occurrence in both breeds. A total of 51 alleles were found (26 for White Fulani and 25 for Muturu). Only six mutual alleles (BoLA-DRB3*048:02, Bo$L A-D R B 3^{*} 011: 02, B o L A-D R B 3^{*} 027: 08, B o L A-D R B 3^{*} 028: 03, B o L A-D R B 3^{*} 087: 02$, $\left.B o L A-D R B 3^{*} 024: 16\right)$ were identified.

Table 1. Allelic polymorphism of BoLA-DRB.3 in White Fulani and Muturu cattle.

\begin{tabular}{|c|c|c|c|}
\hline Allele I. D & Freq. (\%) & Allele I. D & Freq. (\%) \\
\hline White Fulani & & Muturu & \\
\hline$B o L A-D R B 3^{*} 048: 02$ & 5.00 & $B o L A-D R B 3^{*} 048: 02$ & 2.63 \\
\hline$B o L A-D R B 3^{*} 011: 02$ & 10.00 & $B o L A-D R B 3^{*} 011: 02$ & 2.63 \\
\hline$B o L A-D R B 3^{*} 027: 08$ & 2.50 & $B o L A-D R B 3^{*} 027: 08$ & 2.63 \\
\hline$B o L A-D R B 3^{*} 028: 03$ & 7.50 & $B o L A-D R B 3^{*} 028: 03$ & 5.26 \\
\hline$B o L A-D R B 3^{*} 087: 02$ & 2.50 & $B o L A-D R B 3^{*} 087: 02$ & 5.26 \\
\hline$B o L A-D R B 3^{*} 024: 16$ & 2.50 & $B o L A-D R B 3^{*} 024: 16$ & 7.89 \\
\hline BoLA-DRB3 ${ }^{*} 090: 02$ & 2.50 & $B o L A-D R B 3^{*} 083: 01$ & 2.63 \\
\hline$B o L A-D R B 3^{*} 007: 02$ & 2.50 & $B o L A-D R B 3^{*} 024: 02$ & 2.63 \\
\hline$B o L A-D R B 3^{*} 100: 08$ & 2.50 & $B o L A-D R B 3^{*} 087: 04$ & 2.63 \\
\hline$B o L A-D R B 3^{*} 020: 08$ & 2.50 & $B o L A-D R B 3^{*} 011: 01$ & 10.53 \\
\hline$B o L A-D R B 3^{*} 070: 01$ & 5.00 & $B o L A-D R B 3^{*} 027: 10$ & 2.63 \\
\hline$B o L A-D R B 3^{*} 064: 03$ & 5.00 & $B o L A-D R B 3^{*} 011: 05$ & 2.63 \\
\hline$B o L A-D R B 3^{*} 024: 12$ & 2.50 & $B o L A-D R B 3^{*} 021: 01$ & 5.26 \\
\hline$B o L A-D R B 3^{*} 010: 02$ & 2.50 & $B o L A-D R B 3^{*} 009: 02$ & 2.63 \\
\hline$B o L A-D R B 3^{*} 077: 02$ & 2.50 & $B o L A-D R B 3^{*} 020: 10$ & 2.63 \\
\hline$B o L A-D R B 3^{*} 107: 02$ & 2.50 & $B o L A-D R B 3^{*} 005: 06$ & 2.63 \\
\hline$B o L A-D R B 3^{*} 116: 02$ & 2.50 & $B o L A-D R B 3^{*} 061: 01$ & 5.26 \\
\hline$B o L A-D R B 3^{*} 097: 04$ & 2.50 & $B o L A-D R B 3^{*} 024: 07$ & 2.63 \\
\hline$B o L A-D R B 3^{*} 008: 01$ & 7.50 & $B o L A-D R B 3^{*} 020: 14$ & 2.63 \\
\hline$B o L A-D R B 3^{*} 004: 01$ & 5.00 & $B o L A-D R B 3^{*} 024: 20$ & 2.63 \\
\hline BoLA-DRB ${ }^{*} 050: 01: 01$ & 2.50 & $B o L A-D R B 3^{*} 105: 02$ & 2.63 \\
\hline$B o L A-D R B 3^{*} 013: 04$ & 5.00 & $B o L A-D R B 3^{*} 027: 07$ & 10.53 \\
\hline$B o L A-D R B 3^{*} 024: 17$ & 2.50 & $B o L A-D R B 3^{*} 001: 01$ & 5.26 \\
\hline BoLA-DRB3 ${ }^{*} 014: 01: 01$ & 7.50 & $B o L A-D R B 3^{*} 011: 04$ & 2.63 \\
\hline$B o L A-D R B 3^{\star} 138: 01$ & 2.50 & $B o L A-D R B 3^{*} 109: 01$ & 2.63 \\
\hline$B o L A-D R B 3^{*} 087: 01$ & 2.50 & & \\
\hline
\end{tabular}

The boldened first six alleles are mutual between both breeds. Freq. = Frequency. 


\section{Discussion}

\section{BoLA.DRB3.2 alleles in White Fulani and Muturu breeds}

Fifty-one (51) $D R B 3.2$ alleles were identified in total, with only six mutual alleles (BoLA-DRB3 ${ }^{*} 048: 02, \quad B o L A-D R B 3^{*} 011: 02, \quad B o L A-D R B 3^{*} 027: 08, \quad B o-$ $\left.L A-D R B 3^{*} 028: 03, B o L A-D R B 3^{*} 087: 02, B o L A-D R B 3^{*} 024: 16\right)$ between the breeds. This signifies a high within-breed polymorphism and high level of genetic diversity between the two breeds. None of the mutual alleles have been associated with specific disease status. Genetic diversity in BoLA-DRB3.2 between West African zebu and taurine has been documented. Mikko and Anderson [22] found 13 different BRB3.2 alleles in just 18 cattle of White Fulani and N'Dama breed. Alleles with frequency of $5 \%$ and above in this study are BoLA-DRB3 ${ }^{*} 070: 01, B O$ $L A-D R B 3^{*} 014: 01: 01, B o L A-D R B 3^{*} 011: 02, B o L A-D R B 3^{*} 028: 03$,

$B o L A-D R B 3^{*} 008: 01, B o L A-D R B 3^{*} 004: 01$, and BoLA-DRB3*013:04 in White

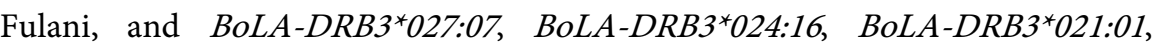
BoLA-DRB3 ${ }^{*} 028: 03, B o L A-D R B 3^{*} 011: 01, B o L A-D R B 3^{*} 087: 02$,

$B o L A-D R B 3^{*} 061: 01$, and BoLA-DRB ${ }^{*} 001: 01$ in Muturu. These alleles ( $\geq 5 \%$ ) accounted for $55 \%$ and $55.25 \%$ of the overall allele frequencies in White Fulani and Muturu breeds, respectively.

BoLA-DRB $3^{*} 070: 01,{ }^{\star} 014: 01: 01$ and ${ }^{*} 008: 01$ which had $\geq 5 \%$ occurrence in White Fulani samples of this study were also identified in White Fulani cattle population studied by Mikko and Anderson [22]. Similarly, BoLA-DRB3*001:01, ${ }^{*} 021: 01$ and ${ }^{*} 011: 01$ that had 5\% frequency in Muturu samples in this study were identified in N'Dama breed, an African taurine like Muturu [22]. When compared with DRB3.2 allele frequencies that have been reported in other Bos indicus breeds, $B o L A-D R B 3^{*} 008: 01$, which is the second most frequently occurring allele in White Fulani population under study (7.5\%), was found as the most frequently occurring allele-23.07\%, $9.2 \%$ and $21.54 \%$ in Caracu [23], Saavedreno Creole [24], and Sistani breeds [25] respectively.

Some prominent alleles identified in this study that have been noted as markers for diseases status and those that occurred more frequently are briefly mentioned below. BoLA-DRB3*014:01:01 allele which occurred at a frequency of $7.5 \%$ in White Fulani cattle population here, was identified in White Fulani, N'Dama and Swedish Red and White European breed [22]. The allele was also found in Latin American Creole cattle (6.64\%), a breed known to have African origin [26]. Takeshima et al. [27] also identified the allele in three South Ameican zebu cattle-Nellore-Brahman (1.54\%), Bolivian Holstein (1.57\%) and Gir (10\%). Takeshima et al. [28] associated BoLA-DRB $3^{*} 014: 01: 01$ allele with Bovine leukemia virus (BLV) resistance in Japanese Holstein cows. This was based on BLV proviral load-an index for BLV diagnosis-in the cows. Alleles Bo$L A-D R B 3^{*} 002: 01,{ }^{*} 009: 02$, and ${ }^{*} 014: 01$ were identified as resistant alleles, while $D R B 3^{*} 012: 01$ and ${ }^{*} 015: 01$ were associated with bovine leukemia virus susceptibility. The allele (BoLA-DRB $\left.3^{*} 014: 01: 01\right)$ was also associated with mastitis resistance in Japanese Holstein [29] [30]. 
$B o L A-D R B 3^{*} 008: 01$ was found in $7.5 \%$ of the White Fulani breed examined. Takeshima et al. [31] identified $B o L A-D R B 3^{\star} 008: 01$ as one of the three most common alleles that accounted for $43.8 \%$ of all the alleles identified in 176 Japanese Shorthorn cattle. BoLA-DRB3 ${ }^{\star} 009: 02$ identified in Muturu population under study, with a frequency of $2.63 \%$, was associated with bovine leukaemia virus resistance [17] [28] [32]. The allele suppressed bovine leukaemia virus replication in Japanese Black and Holstein breed in Japan [32].

Gutiérrez et al. [33] reported that $B O L A-D R B 3^{*} 011: 01$, which was found in Muturu population in this study (10.53\% frequency) confers resistance to bovine leukaemia in Harton del Valle cattle breed, with heterozygous individuals responding better to the disease than the homozygous carriers. $B o L A-D R B 3^{\star} 001: 01$ (5.26\%) and $B o L A-D R B 3^{\star} 011: 01$ (10.53\%) identified in Muturu breed were associated with susceptibility and resistance to mastitis respectively in Japanese Holstein [29] [30].

Peters et al. [34] investigated BoLA-DRB3.2 genetic diversity in 17 cattle breeds from Africa, Asia, and America. Their result showed a higher within breed genetic variation than between breed variation. Three Nigerian breeds (White Fulani, Muturu, and Sokoto Gudali) were included in their study. Sokoto Gudali had 10 haplotypes, which ranked as the highest number of haplotypes observed in a single breed in the study. Muturu and White Fulani had six and eight haplotypes, respectively. This result gave an insight into how polymorphic the Muturu and White Fulani breeds are at the BoLA-DRB3.2 locus.

The allelic information provided in this study would add to the pool of knowledge currently available on DRB3.2 alleles in Nigerian cattle breeds and could also be useful for subsequent association with infectious diseases and immunological traits. It should be noted that the alleles ascribed to each breed here were those with the highest percentage identity with the $D R B 3.2$ sequence for each animal.

A better method to identify $B O L A-D R B 3$ alleles in the highly polymorphic exon two regions would be to use next-generation sequencing, as opposed to Sanger sequencing used here. This would reveal haplotypes and zygosity status for each animal, thereby providing enough confidence to assert the presence of novel alleles.

\section{Conclusion}

$B o L A-D R B 3.2$ locus appears to be very polymorphic in White Fulani and $\mathrm{Mu}-$ turu cattle breeds. BoLA-DRB3.2 alleles found in both breeds were identified, some of which have been associated with disease resistance/susceptibility in other cattle breeds. It will be worthy to further explore these alleles in a bid to reveal their status on disease resistance/susceptibility in White Fulani and Muturu cattle.

\section{Authors Contribution}

MJ Hegarty supervised the whole process. Ridwan Olawale Ahmed designed the 
experiment, carried out laboratory works and wrote the manuscript. Semiu Folaniyi Bello collected field data-collection of nasal swabs. All authors read and approved the manuscript.

\section{Conflicts of Interest}

The authors declare no conflicts of interest regarding the publication of this paper.

\section{References}

[1] Edwards, S.V. and Hedrick, P.W. (1998) Evolution and Ecology of MHC Molecules: From Genomics to Sexual Selection. Trends in Ecology \& Evolution, 13, 305-311. https://doi.org/10.1016/S0169-5347(98)01416-5

[2] Hill, A.V. (1998) The Immunogenetics of Human Infectious Diseases. Annual Review of Immunology, 16, 593-617. https://doi.org/10.1146/annurev.immunol.16.1.593

[3] Ellis, S.A. and Ballingall, K.T. (1999) Cattle MHC: Evolution in Action? Immunological Reviews, 167, 159-168. https://doi.org/10.1111/j.1600-065X.1999.tb01389.x

[4] Fries, R., Hediger, R. and Stranzinger, G. (1986) Tentative Chromosomal Localization of the Bovine Major Histocompatibility Complex by in Situ Hybridization. Animal Genetics, 17, 287-294. https://doi.org/10.1111/j.1365-2052.1986.tb00722.x

[5] Behl, J.D., Verma, N.K., Tyagi, N., Mishra, P., Behl, R. and Joshi, B.K. (2012) The Major Histocompatibility Complex in Bovines: A Review. International Scholarly Research Notices, 2012, Article ID: 872710. https://doi.org/10.5402/2012/872710

[6] Takeshima, S.N. and Aida, Y. (2006) Structure, Function and Disease Susceptibility of the Bovine Major Histocompatibility Complex. Animal Science Journal, 77, 138-150. https://doi.org/10.1111/j.1740-0929.2006.00332.x

[7] Behl, J.D., Verma, N.K., Behl, R., Mukesh, M. and Ahlawat, S.P.S. (2007) Characterization of Genetic Polymorphism of the Bovine Lymphocyte Antigen DRB3.2 Locus in Kankrej Cattle (Bos indicus). Journal of Dairy Science, 90, 2997-3001. https://doi.org/10.3168/jds.2006-547

[8] Sun, L., Song, Y., Riaz, H. and Yang, L. (2013) Effect of BoLA-DRB3 Exon2 Polymorphisms on Lameness of Chinese Holstein Cows. Molecular Biology Reports, 40, 1081-1086. https://doi.org/10.1007/s11033-012-2150-6

[9] Miyasaka, T., Takeshima, S.N., Matsumoto, Y., Kobayashi, N., Matsuhashi, T., Miyazaki, Y., et al. (2011) The Diversity of Bovine MHC Class II DRB3 and DQA1 Alleles in Different Herds of Japanese Black and Holstein Cattle in Japan. Gene, 472, 42-49. https://doi.org/10.1016/j.gene.2010.10.007

[10] Da Mota, A.F., Martinez, M.L. and Coutinho, L.L. (2004) Genotyping BoLA-DRB3 Alleles in Brazilian Dairy Gir Cattle (Bos indicus) by Temperature-Gradient Gel Electrophoresis (TGGE) and Direct Sequencing. European Journal of Immunogenetics, 31, 31-35. https://doi.org/10.1111/j.1365-2370.2004.00438.x

[11] Martinez, M.L., Machado, M.A., Nascimento, C.S., Silva, M.V.G.B., Teodoro, R.L., Furlong, J., et al. (2006) Association of BoLA-DRB3.2 Alleles with Tick (Boophilus microplus) Resistance in Cattle. Genetics and Molecular Research, 5, 513-524.

[12] Rupp, R., Hernandez, A. and Mallard, B.A. (2007) Association of Bovine Leukocyte Antigen (BoLA) DRB3.2 with Immune Response, Mastitis, and Production and Type Traits in Canadian Holsteins. Journal of Dairy Science, 90, 1029-1038. 
https://doi.org/10.3168/jds.S0022-0302(07)71589-8

[13] Juliarena, M.A., Poli, M., Sala, L., Ceriani, C., Gutierrez, S., Dolcini, G., et al. (2008) Association of BLV Infection Profiles with Alleles of the BoLA-DRB3.2 Gene. Animal Genetics, 39, 432-438. https://doi.org/10.1111/j.1365-2052.2008.01750.x

[14] Starkenburg, R.J., Hansen, L.B., Kehrli Jr., M.E. and Chester-Jones, H. (1997) Frequencies and Effects of Alternative DRB3.2 Alleles of Bovine Lymphocyte Antigen for Holsteins in Milk Selection and Control Lines. Journal of Dairy Science, 80, 3411-3419. https://doi.org/10.3168/jds.S0022-0302(97)76316-1

[15] Nascimento, C.S.D., Machado, M.A., Martinez, M.L., Silva, M.V.G., Guimarães, M.F.M., Campos, A.L., et al. (2006) Association of the Bovine Major Histocompatibility Complex (BoLA) BoLA-DRB3 Gene with Fat and Protein Production and Somatic Cell Score in Brazilian Gyr Dairy Cattle (Bos indicus). Genetics and Molecular Biology, 29, 641-647. https://doi.org/10.1590/S1415-47572006000400011

[16] Takeshima, S.N., Miyasaka, T., Polat, M., Kikuya, M., Matsumoto, Y., Mingala, C.N., et al. (2014) The Great Diversity of Major Histocompatibility Complex Class II Genes in Philippine Native Cattle. Meta Gene, 2, 176-190. https://doi.org/10.1016/j.mgene.2013.12.005

[17] Carignano, H.A., Beribe, M.J., Caffaro, M.E., Amadio, A., Nani, J.P., Gutierrez, G., et al. (2017) BOLA-DRB3 Gene Polymorphisms Influence Bovine Leukaemia Virus Infection Levels in Holstein and Holstein $\times$ Jersey Crossbreed Dairy Cattle. Animal Genetics, 48, 420-430. https://doi.org/10.1111/age.12566

[18] Juliarena, M.A., Lendez, P.A., Gutierrez, S.E., Forletti, A., Rensetti, D.E. and Ceriani, M.C. (2013) Partial Molecular Characterization of Different Proviral Strains of Bovine Leukemia Virus. Archives of Virology, 158, 63-70. https://doi.org/10.1007/s00705-012-1459-8

[19] Ahmed, R.O., Bello, S.F., Shu'aibu, I. and Hegarty, M.J. (2020) An Investigation of Polymorphism in SMO and LMF1 Genes and Their Association with Body Size in African White Fulani and Muturu Cattle Breeds. Advances in Bioscience and Biotechnology, 11, 319-344. https://doi.org/10.4236/abb.2020.117023

[20] Baxter, R., Hastings, N., Law, A. and Glass, E.J. (2008) A Rapid and Robust Sequence-Based Genotyping Method for BoLA-DRB3 Alleles in Large Numbers of Heterozygous Cattle. Animal Genetics, 39, 561-563.

https://doi.org/10.1111/j.1365-2052.2008.01757.x

[21] Miltiadou, D., Law, A.S. and Russell, G.C. (2003) Establishment of a Sequence-Based Typing System for BoLA-DRB3 Exon 2. Tissue Antigens, 62, 55-65. https://doi.org/10.1034/j.1399-0039.2003.00080.x

[22] Mikko, S. and Anderson, L. (1995) Extensive MHC Class II DRB3 Diversity in African and European Cattle. Immunogenetics, 42, 408-403.

https://doi.org/10.1007/BF00179403

[23] Miretti, M.M., Ferro, J.A., Lara, M.A. and Contel, E.P.B. (2001) Restriction Fragment Length Polymorphism (RFLP) in Exon 2 of the BoLA-DRB3 Gene in South American Cattle. Biochemical Genetics, 39, 311-324.

https://doi.org/10.1023/A:1012204829894

[24] Ripoli, M.V., Liron, J.P., De Luca, J.C., Rojas, F., Dulout, F.N. and Giovambattista, G. (2004) Gene Frequency Distribution of the BoLA-DRB3 Locus in Saavedreño Creole Dairy Cattle. Biochemical Genetics, 42, 231-240. https://doi.org/10.1023/B:BIGI.0000034427.66887.fe

[25] Mohammadi, A., Nassiry, M.R., Mosafer, J., Mohammadabadi, M.R. and Sulimova, G.E. (2009) Distribution of BoLA-DRB3 Allelic Frequencies and Identification of a 
New Allele in the Iranian Cattle Breed Sistani (Bos indicus). Russian Journal of Genetics, 45, 198-202. https://doi.org/10.1134/S1022795409020100

[26] Giovambattista, G., Takeshima, S.N., Ripoli, M.V., Matsumoto, Y., Franco, L.A.A., Saito, H., et al. (2013) Characterization of Bovine MHC DRB3 Diversity in Latin American Creole Cattle Breeds. Gene, 519, 150-158. https://doi.org/10.1016/j.gene.2013.01.002

[27] Takeshima, S.N., Corbi-Botto, C., Giovambattista, G. and Aida, Y. (2018) Genetic Diversity of BoLA-DRB3 in South American Zebu Cattle Populations. BMC Genetics, 19, 33. https://doi.org/10.1186/s12863-018-0618-7

[28] Takeshima, S.N., Ohno, A. and Aida, Y. (2019) Bovine Leukemia Virus Proviral Load Is More Strongly Associated with Bovine Major Histocompatibility Complex Class II DRB3 Polymorphism than with DQA1 Polymorphism in Holstein Cow in Japan. Retrovirology, 16, 14.

[29] Yoshida, T., Mukoyama, H., Furuta, H., Kondo, Y., Takeshima, S.N., Aida, Y., et al. (2009) Association of BoLA-DRB3 Alleles Identified by a Sequence-Based Typing Method with Mastitis Pathogens in Japanese Holstein Cows. Animal Science Journal, 80, 498-509. https://doi.org/10.1111/j.1740-0929.2009.00663.x

[30] Yoshida, T., Mukoyama, H., Furuta, H., Kondo, Y., Takeshima, S.N., Aida, Y., et al. (2009) Association of the Amino Acid Motifs of BoLA-DRB3 Alleles with Mastitis Pathogens in Japanese Holstein Cows. Animal Science Journal, 80, 510-519. https://doi.org/10.1111/j.1740-0929.2009.00664.x

[31] Takeshima, S., Nakai, Y., Ohta, M. and Aida, Y. (2002) Characterization of DRB3 Alleles in the MHC of Japanese Shorthorn Cattle by Polymerase Chain Reaction-Sequence-Based Typing. Journal of Dairy Science, 85, 1630-1632. https://doi.org/10.3168/jds.S0022-0302(02)74234-3

[32] Hayashi, T., Mekata, H., Sekiguchi, S., Kirino, Y., Mitoma, S., Honkawa, K., et al. (2017) Cattle with the BoLA Class II $D R B 3^{*} 0902$ Allele Have Significantly Lower Bovine Leukemia Proviral Loads. Journal of Veterinary Medical Science, 79, 1552-1555. https://doi.org/10.1292/jvms.16-0601

[33] Gutiérrez, S.E., Esteban, E.N., Lützelschwab, C.M. and Juliarena, M.A. (2017) Major Histocompatibility Complex-Associated Resistance to Infectious Diseases: The Case of Bovine Leukemia Virus Infection. Trends and Advances in Veterinary Genetics, 6, 101-126. https://doi.org/10.5772/65804

[34] Peters, S.O., Hussain, T., Adenaike, A.S., Adeleke, M.A., De Donato, M., Hazzard, J., et al. (2018) Genetic Diversity of Bovine Major Histocompatibility Complex Class II DRB3 Locus in Cattle Breeds from Asia Compared to Those from Africa and America. Journal of Genomics, 6, 88-97. https://doi.org/10.7150/jgen.26491 
Appendix. BoLA-DRB3.2 Alleles, Percentage Identity and Hit Length

\begin{tabular}{|c|c|c|c|c|c|c|}
\hline & \multicolumn{3}{|c|}{ White Fulani } & \multicolumn{3}{|c|}{ Muturu } \\
\hline & Allele & \%I.D & $\mathrm{HL}$ & Allele & \%I.D & HL \\
\hline 1 & BoLA-DRB3*010:02 & 99.63 & 273 & BoLA-DRB3*027:07 & 99.20 & 269 \\
\hline 2 & BoLA-DRB3*070:01 & 93.63 & 270 & BoLA-DRB3*011:04 & 96.83 & 269 \\
\hline 3 & BoLA-DRB3*007:02 & 95.17 & 272 & BoLA-DRB3*020:14 & 97.68 & 261 \\
\hline 4 & BoLA-DRB3*116:02 $^{*}$ & 96.55 & 263 & BoLA-DRB3*024:16 & 97.73 & 267 \\
\hline 5 & BoLA-DRB3*014:01:01 & 92.58 & 269 & BoLA-DRB3*024:16 & 96.63 & 270 \\
\hline 6 & BoLA-DRB3*107:02 & 90.07 & 267 & BoLA-DRB3*021:01 & 97.20 & 269 \\
\hline 7 & BoLA-DRB3*090:02 & 98.12 & 270 & BoLA-DRB3 ${ }^{*} 028: 03$ & 97.39 & 276 \\
\hline 8 & BoLA-DRB3*027:08 & 96.44 & 274 & BoLA-DRB3*083:01 & 95.85 & 268 \\
\hline 9 & BoLA-DRB3*100:08 & 95.45 & 271 & BoLA-DRB3*024:02 & 96.67 & 270 \\
\hline 10 & BoLA-DRB3*020:08 & 95.65 & 259 & BoLA-DRB3*087:04 & 93.50 & 271 \\
\hline 11 & BoLA-DRB3*070:01 & 90.84 & 271 & BoLA-DRB3*027:07 & 96.80 & 271 \\
\hline 12 & BoLA-DRB3*011:02 & 98.41 & 275 & BoLA-DRB3*027:07 & 100.00 & 274 \\
\hline 13 & BoLA-DRB3 ${ }^{\star} 064: 03$ & 96.62 & 272 & BoLA-DRB3*011:01 & 96.24 & 268 \\
\hline 14 & BoLA-DRB3*024:12 & 97.69 & 263 & BoLA-DRB3*027:10 & 96.50 & 260 \\
\hline 15 & BoLA-DRB3*048:02 & 96.58 & 268 & BoLA-DRB3*024:16 & 97.75 & 270 \\
\hline 16 & BoLA-DRB3*011:02 & 97.70 & 267 & BoLA-DRB3*011:05 & 96.98 & 268 \\
\hline 17 & BoLA-DRB3*014:01:01 & 99.18 & 244 & BoLA-DRB3*087:02 & 97.75 & 270 \\
\hline 18 & BoLA-DRB3077:02 & 97.72 & 263 & BoLA-DRB3*009:02 & 99.62 & 268 \\
\hline 19 & BoLA-DRB3 ${ }^{*} 024: 16$ & 98.12 & 269 & BoLA-DRB3 ${ }^{*} 021: 01$ & 96.25 & 273 \\
\hline 20 & BoLA-DRB3028:03 & 98.00 & 255 & BoLA-DRB3 ${ }^{*} 020: 10$ & 98.80 & 270 \\
\hline 21 & BoLA-DRB3028:03 & 97.62 & 270 & BoLA-DRB3 ${ }^{*} 027: 07$ & 100.00 & 268 \\
\hline 22 & BoLA-DRB3*097:04 & 97.72 & 270 & BoLA-DRB3*005:06 & 97.00 & 269 \\
\hline 23 & BoLA-DRB3*028:03 & 96.59 & 268 & BoLA-DRB3*087:02 & 97.14 & 261 \\
\hline 24 & BoLA-DRB3*011:02 & 97.69 & 266 & BoLA-DRB3*061:01 & 95.90 & 267 \\
\hline 25 & BoLA-DRB3*011:02 & 97.25 & 274 & BoLA-DRB3*011:01 & 98.39 & 268 \\
\hline 26 & BoLA-DRB3*008:01 & 97.60 & 268 & BoLA-DRB3 ${ }^{*} 011: 01$ & 99.20 & 268 \\
\hline 27 & BoLA-DRB3*008:01 & 97.74 & 267 & BoLA-DRB3*024:07 & 98.15 & 270 \\
\hline 28 & BoLA-DRB3 ${ }^{*} 004: 01$ & 97.03 & 274 & BoLA-DRB3 ${ }^{*} 027: 08$ & 97.18 & 253 \\
\hline 29 & BoLA-DRB3*048:02 & 98.39 & 268 & BoLA-DRB3*024:20 & 97.36 & 272 \\
\hline 30 & BoLA-DRB3*014:01:01 & 96.46 & 266 & BoLA-DRB3*011:01 & 98.87 & 278 \\
\hline 31 & BoLA-DRB3*050:01:01 & 95.88 & 67 & BoLA-DRB3*105:02 & 98.71 & 273 \\
\hline 32 & BoLA-DRB3*013:04 & 98.08 & 267 & BoLA-DRB3*048:02 & 99.25 & 272 \\
\hline 33 & BoLA-DRB3*024:17 & 97.36 & 268 & BoLA-DRB3 ${ }^{*} 001: 01$ & 95.88 & 274 \\
\hline 34 & BoLA-DRB3013:04 & 98.11 & 273 & BoLA-DRB3*028:03 & 97.00 & 267 \\
\hline 35 & BoLA-DRB3*087:02 & 96.63 & 269 & BoLA-DRB3*061:01 & 96.00 & 274 \\
\hline 36 & BoLA-DRB3*064:03 & 96.67 & 276 & BoLA-DRB3*011:02 & 97.22 & 268 \\
\hline 37 & BoLA-DRB3*004:01 & 97.00 & 267 & BoLA-DRB3*001:01 & 98.18 & 274 \\
\hline 38 & BoLA-DRB3*008:01 & 96.27 & 273 & BoLA-DRB3*109:01 & 97.25 & 270 \\
\hline 39 & BoLA-DRB3*138:01 & 97.36 & 267 & & & \\
\hline 40 & BoLA-DRB3*087:01 & 97.38 & 274 & & & \\
\hline
\end{tabular}

\%I.D—\% Identity, HL—Hit length. 